\title{
Vemurafenib provides a rapid and robust clinical response in paediatric Langerhans cell histiocytosis with the BRAF V600E mutation but does not eliminate low-level minimal residual disease based on ddPCR using cell-free circulating DNA
}

\author{
Dmitry Evseev ${ }^{1}$, Irina Kalinina ${ }^{1}$, Elena Raykina ${ }^{1}$, Daria Osipova ${ }^{1}$, Zalina Abashidze ${ }^{1}$, \\ Anna Ignatova ${ }^{1}$, Anna Mitrofanova ${ }^{1}$, Alexei Maschan ${ }^{1}$, Galina Novichkova ${ }^{1}$, and Michael \\ Maschan $^{1}$ \\ ${ }^{1}$ Dmitry Rogachev National Medical Research Center of Pediatric Hematology Oncology \\ and Immunology
}

December 3, 2020

\begin{abstract}
Background Langerhans cell histiocytosis (LCH) involves abnormal proliferation of Langerhans cells (LC), which is typically driven by the BRAF V600E mutation. High-risk LCH has a poor prognosis. Procedure Fifteen children (5 girls, 10 boys) with BRAF V600E + LCH received vemurafenib (initial dose median $40 \mathrm{mg} / \mathrm{kg} /$ day, range: 11-51.6 mg/ $\mathrm{kg} / \mathrm{day}$ ) between March 2016 and February 2020. All patients had previous received LCH-directed chemotherapy. The median age at LCH onset was 2 months (range: 1-28 months) and the median age at the start of vemurafenib treatment was 22 months (range: 13-62 months). The median disease activity score (DAS) at the start of vemurafenib treatment was 12 points (range: 2-22 points). Results The median duration of vemurafenib therapy was 29 months (range: $2.4-45$ months). All patients responded to treatment, with median DAS values of 4 points (range: 0-14 points) at week 4 and 1 point (range: 0-3 points) at week 26. Toxicities included skin/hair changes (93\%) and non-significant QT prolongation (73\%). Two patients died, including 1 patient who experienced hepatic failure after NSAID overdose and 1 patient who developed neutropenic sepsis. Electively stopping vemurafenib treatment resulted in relapse in 5 patients, and complete cessation was only possible for 1 patient. Digital droplet PCR for BRAF V600E using cell-free circulating DNA revealed that 7 patients had mutation statuses that fluctuated over time. Conclusion Our study confirms that vemurafenib treatment is safe and effective for young children with BRAF V600E+ multisystem LCH. However, treatment using vemurafenib does not completely eliminate the disease.
\end{abstract}

\section{Hosted file}

Vemurafenib our experience v5.4 PBC.pdf available at https://authorea.com/users/381037/ articles/496841-vemurafenib-provides-a-rapid-and-robust-clinical-response-in-paediatriclangerhans-cell-histiocytosis-with-the-braf-v600e-mutation-but-does-not-eliminate-lowlevel-minimal-residual-disease-based-on-ddpcr-using-cell-free-circulating-dna

\section{Hosted file}

Table 1.pdf available at https://authorea.com/users/381037/articles/496841-vemurafenibprovides-a-rapid-and-robust-clinical-response-in-paediatric-langerhans-cellhistiocytosis-with-the-braf-v600e-mutation-but-does-not-eliminate-low-level-minimalresidual-disease-based-on-ddpcr-using-cell-free-circulating-dna 

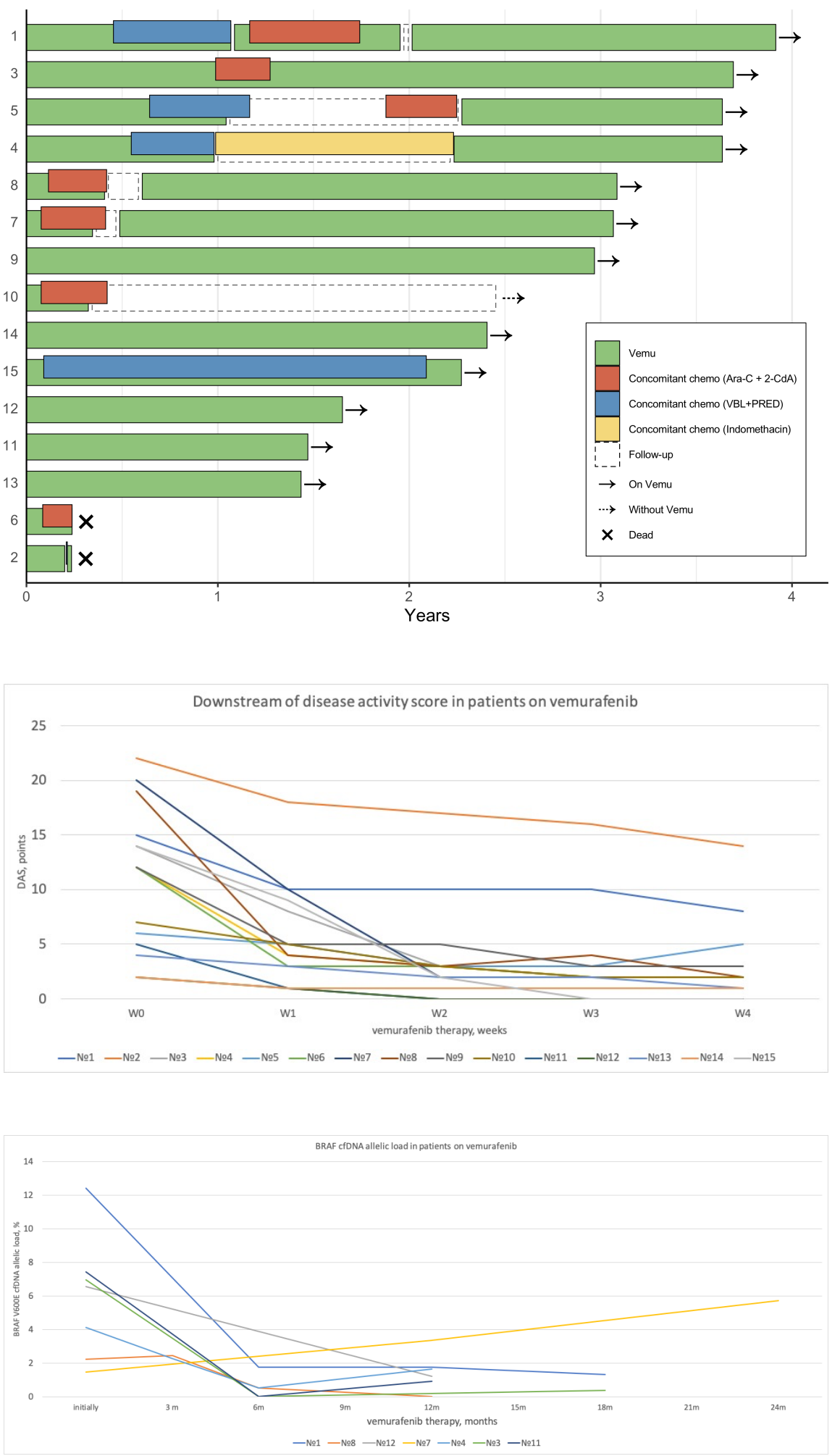